\title{
Beyond Conventional 3D Ultrafast Laser Material Processing
}

\author{
P. G. Kazansky ${ }^{l}$, M. Beresna ${ }^{l}$ and Y. Bellouard ${ }^{2}$ \\ 1. Optoelectronics Research Centre, University of Southampton, SO171BJ Southampton, UK \\ 2. Department of Mechanical Engineering, Eindhoven University of Technology, 5600 MB Eindhoven, The Netherlands
}

Material processing with ultrafast lasers has attracted considerable interest due to a wide range of applications from laser surgery and integrated optics to optical data storage, 3D micro- and nano-structuring $[1,2]$. A decade ago it has been discovered that under certain irradiation conditions ordered sub-wavelength structures with features smaller than $20 \mathrm{~nm}$ can be formed in the volume of silica glass [3]. The effect of nanograting formation has attracted considerable interest with proposals of applications ranging from nanofluidics [4,5] to polarization control devices [6].

More recently, the self-assembled sub-wavelength nanostructuring have been proposed for fabrication of vortex polarization converters and rewritable polarization multiplexed optical memory [7], where the information encoding is realized by means of two birefringence parameters, i.e. the slow axis orientation (4th dimension) and retardance (5th dimension), in addition to three spatial coordinates (Fig.1).

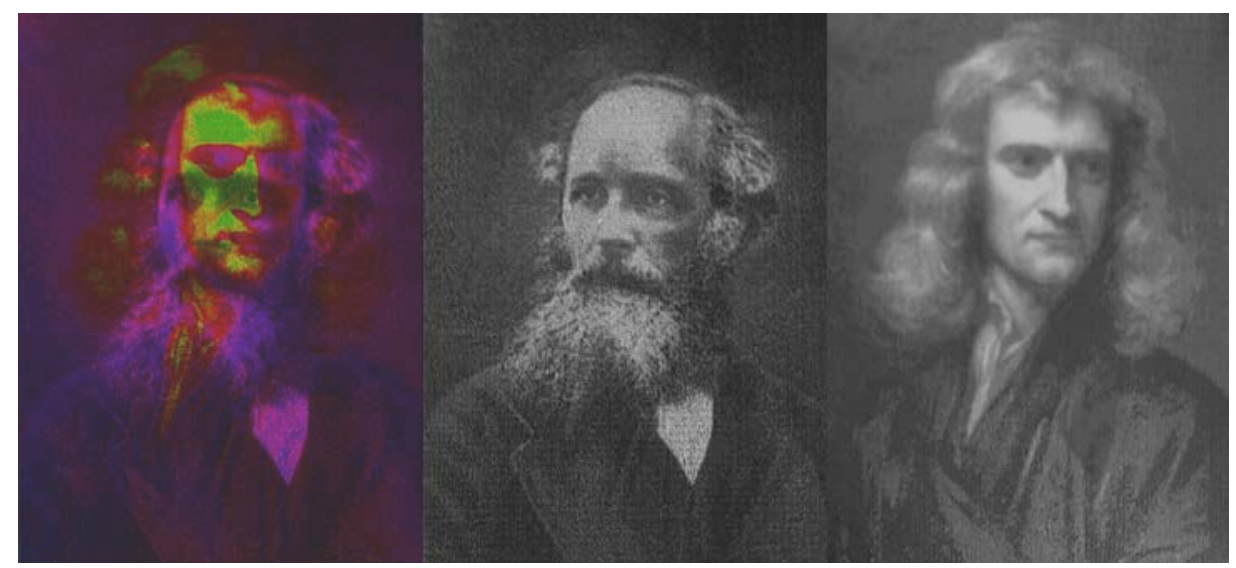

Fig. 1 | Ultrafast optical recording via self-assembled nanograting induced birefringence in fused silica. Maxwell and Newton are recorded in one image (left, in pseudo colours), however, they can be easily decoupled as Maxwell is recorded in strength of retardance (centre) and Newton in azimuth of the slow axis (right). Size is $1.5 \times 2 \mathrm{~mm}$.

A remarkable effect has also been discovered, referred to as quill or calligraphic laser writing, which reveals strong dependence of the material modification, in particular the self-assembled sub-wavelength structures in glass, on orientation of the writing direction relative to direction of the pulse front tilt [8-10]. Moreover, evidence of the first order phase transition associated with self-assembled nanostructures formation was revealed and supercooled state of laser damage was observed using pulses with tilted intensity front. More recently it has been demonstrated that the tip of an ultrafast laser quill has a property that is very different from an ordinary quill [11]. Specifically, the modification of glass can be controlled even in stationary conditions by the mutual orientation of light polarization azimuth and the pulse front tilt. Figuratively, the polarization can be used as a sharpening blade for the ultrafast light quill. The demonstrations of self-assembled nano-structuring and employing mutual orientations of beam movement or the light polarization plane and pulse front tilt to control interaction of matter with ultrashort light pulses, open new opportunities in material processing.

\section{References}

[1] R. R. Gattas and E. Mazur, "Femtosecond laser micromachining in transparent materials," Nature Photonics 2, 219 (2008).

[2] W. Yang, P. G. Kazansky, and Yu. P. Svirko, "Non-reciprocal ultrafast laser writing," Nature Photonics 2, 99 (2008).

[3] Y. Shimotsuma, P. G. Kazansky, J. Qiu, and K. Hirao, "Self-organized nanogratings in glass irradiated by ultrashort light pulses," Phys. Rev. Lett. 91, 247705 (2003).

[4] Y. Bellouard, A. Said, M. Dugan, and P. Bado, "Fabrication of high-aspect ratio, micro-fluidic channels and tunnels using femtosecond laser pulses and chemical etching," Opt. Express 12, 2120 (2004).

[5] V. Bhardwaj, E. Simova, P. Rajeev, C. Hnatovsky, R. Taylor, D. Rayner, and P. Corkum, "Optically produced arrays of planar nanostructures inside fused silica," Phys. Rev. Lett. 96, 057404-1 (2006).

[6] M. Beresna and P. G. Kazansky, "Polarization diffraction grating produced by femtosecond laser nanostructuring in glass," Opt. Lett. 35, $1662(2010)$.

[7] Y. Shimotsuma et. al., "Ultrafast manipulation of self-assembled form birefringence in glass,"Advanced Materials 22 (36), 4039 (2010).

[8] P. G. Kazansky et al., "Quill" writing with ultrashort light pulses in transparent materials," Appl. Phys. Lett. 90, 151120 (2007).

[9] B. Poumellec et al., "Non reciprocal writing and chirality in femtosecond laser irradiated silica," Opt. Express 16, 18354 (2008).

[10]D. N. Vitek, E. Block, Y. Bellouard, D. E. Adams, S. Backus, D. Kleinfeld, C. G. Durfee, and J. A. Squier, "Spatio-temporally focused femtosecond laser pulses for nonreciprocal writing in optically transparent materials," Opt. Express 18, 24673 (2010).

[11]P. G. Kazansky et al., "Polarization control of ultrafast laser writing using pulses with tilted front", submitted to Phys. Rev. Lett. (2011). 